Pirineos, 145-146: 65 a 8O, JACA; 1995

\title{
CICLOS BIOGEOQUÍMICOS EN BOSQUES DE LA SIERRA DE BÉJAR (SALAMANCA). RETORNO DE bioelementos POR MEDIO DEL AGUA DE LLUVIA
}

\author{
I. SANTA REgina
}

Instituto de Recursos Naturales y Agrobiología. Apdo. 257. 3707.1 Salamanca

\begin{abstract}
RESUMEN. - Se ha estimado el retorno anual de bioelementos a través del agua de lluvia en un bosque de repoblación de pino albar (Pinus sylvestris L.) en la Cuenca de Candelario (sistema Central).

Del total de elementos aportados al suelo, la inmensa mayoría del carbono, nitrógeno, fósforo, calcio, potasio, sodio y zinc, penetran por medio del agua de pluviolavado, sólo un $10 \%$ lo hace, a través del agua de escurrimiento fustal; el magnesio alcanza el suelo en un $82 \%$ por el agua de pluviolavado y el resto por escurrimiento fustal; el manganeso y el hierro 10 hacen en un $70 \%$ y $30 \%$ respectivamente, mientras que el aporte predominante de cobre se realiza a través del agua de escurrimiento fustal (más del 60\%).

ABSTRACT.- The annual return of bioelements by rainfall was estimated in afforested areas of scots pine (Pinus sylvestris L.) in. the Candelario Basin (Central System, Spain).

Of the total elements contributed to the soil in rainwater, most of the carbon, nitrogen, phosphorus, calcium, potassium, sodium and zinc enter the soil via throughfall, while only $10 \%$ do so through stemflow; $82 \%$ of the magnesium reaches the soil via throughfall and the rest by stemflow: manganese and iron do so at percentages of 70 and $30 \%$ respectively, while the main contribution of copper is via stemflow (more than 60\%).
\end{abstract}

Key words: Biogeochemicalcycles, throughfall, stemflow, Pinus sylvestris, L., Sierra de Béjar.

\section{Introducción}

El agua de lluvia que cae sobre las hojas se enriquece en materia orgánica y elementos minerales; los productos excretados por las hojas son, en efecto, arrastrados por el agua, así como una parte importante de la microflora (Diem, 1973), además de polvo atmosférico pegado sobre las hojas. Tukey (1966) ha estudiado el mecanismo de este fenómeno de secreción cuticular, que depende de la edad de las hojas, de las especies 
vegetales y, también, de las variedades, aunque sólo hay escasos estudios sobre el aspecto cualitativo y cuantitativo de los aportes de materia orgánica por pluviolavados.

El agua que fluye por las ramas y troncos confiere ciertas características importantes a lazona cercana al árbol y ha sido un tema abordado por diversos autores (Slatyer, 1962).

Dos explicaciones han sido dadas al origen de los cationes pluviolavados (Rapp, 1971): la primera, atribuye los elementos pluviolavados a la vegetación misma, siendo la teoría de la secreción de sales minerales; la segunda, limita la acción de la vegetación a un papel intermediario (responsable de la fijación de aerosoles y de polvos atmosféricos, los cuales serán arrastrados posteriormente por las precipitaciones).

El máximo enriquecimiento de las aguas de lluvia (Rapp, 1971) tiene lugar en el momento de la formación de nuevas hojas y caída de las viejas. Durante este período, la cutícula poco espesa en las hojas más jóvenes puede permitir una secreción más importante; las acículas viejas tienen un metabolismo ralentizado antes de soltarse y liberan más fácilmente sus elementos constitutivos.

Diversos autores han evidenciado la distribución del agua de lluvia sobre los ecosistemas forestales y el aporte de bioelementos que llega al suelo de dichos bosques. Destacan los estudios de Rutter (1963), Patric (1966), Santa Regina (1987), Santa Regina \& Gallardo (1989) y Santa Regina et al. (1989) en poblaciones de coníferas; Slavik (1962) y Schnock y Galoux (1967) en fagáceas; y Jackson (1975), en bosques tropicales.

El objetivo del presente trabajo es conocer el aporte de elementos minerales al substrato edáfico del pinar por medio del agua de lluvia y los procesos que conlleva su paso por la canopia vegetal, para una mejor comprensión del reciclaje de elementos totales en la zona de estudio.

\section{Material y métodos}

\subsection{Descripción de la zona de estudio}

La zona de estudio se encuentra en la vertiente Norte de la Sierra de Béjar (Provincia de Salamanca, España) (Fig. 1), y posee una cubierta vegetal climácica de Quercus pyrenaica sobre cambisoles húmicos (F.A.O., 1973); este bosque se ha sustituido por pinares.

El pinar de repoblación seleccionado data del año 1955, sin aclareo posterior, salvo alguna poda de ramas bajas. Dada la alta densidad del arbolado, se observan ejemplares muertos por la carencia de luz solar, debido a la fuerte competencia en altura. Se encuentra a $1550 \mathrm{~m}$ de altitud, en un camino forestal secundario que se dirige hacia el límite provincial, tras atravesar el torrente de Peñas Gordas. Se ha cercado un área de 340 $\mathrm{m}^{2}$, habiéndose instalado también recipientes de medida de aguas de lluvia (incidente y pluviolavado) y escurrimiento fustal. 


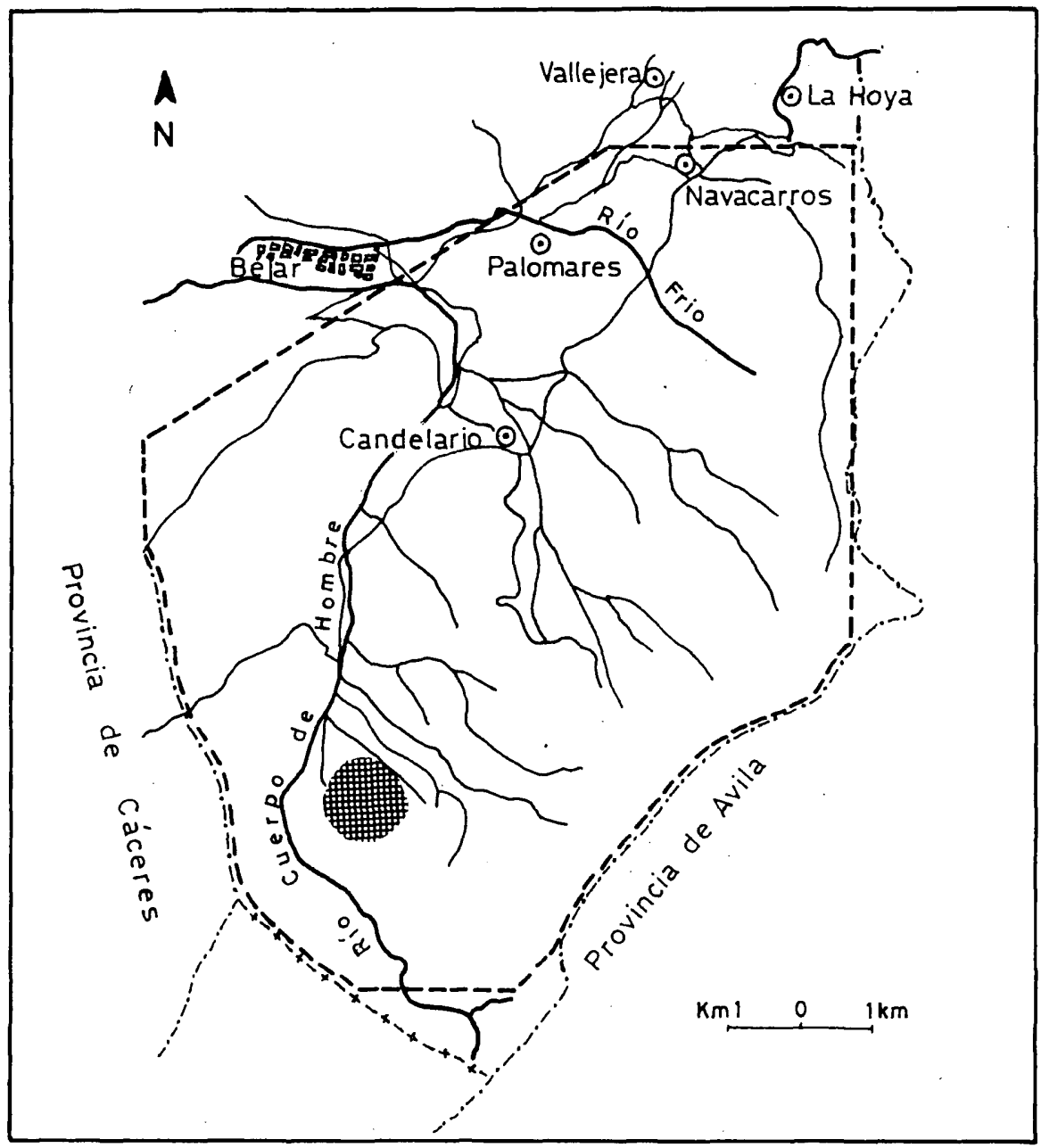

Fig. 1. Situación de la zona de estudio.

En cuanto al suelo, se trata de una asociación de Rankers y Cambisoles húmicos, dada la diferenciación lateral de profundidad observable, que se desarrollan sobre granito alterado (fase delgada) y arena granítica (fase profunda), con pedregosidad variable.

En el suelo del pinar se observa la ausencia casi completa de fanerógamas debido a los productos fenólicos que aportan las resinosas al estrato inferior (mantillo), acidificándose éste; además, la descomposición lenta de los restos orgánicos, con acumulación de hojarasca, origina condicio- 
nes físicas y fisicoquímicas poco favorables para el desarrollo herbáceo (Santa Regina, 1987).

El estrato arbóreo está dominado por fustes de Pinus sylvestris; su densidad media estimada oscila alrededor de los 1700 árboles/ha; su diámetro medio, a 1,5 $\mathrm{m}$ de altura, es de $19,8 \mathrm{~cm}$, siendo el crecimiento anual medio calculado de $1,4 \mathrm{~cm}$; la altura media estimada es de unos $9 \mathrm{~m}$.

El estrato arbustivo es escaso y dominado por Genista florida. En el sotobosque, las fanerógamas de porte herbáceo más abundantes son: Leucanthemopsis flaveola, Cerastium glomeratum, C. brachipetalum, Veronica hederaefolia, Tesdalia nudicaulis, Raununculus bulbosus var. alleae y Arenaria montana. (Santa Regina, 1987).

En cuanto a la climatología se hace referencia a datos de precipitación de 14 años de una estación pluviométrica que existió en Béjar y de termometría durante 20 años en Barco de Avila, corregidos para la zona de Béjar. La representación del diagrama ombrotérmico de WalterGaussen (Fig. 2) muestra que es un ombroclima subhúmedo; sin embar-

BEJAR (Salamanca)

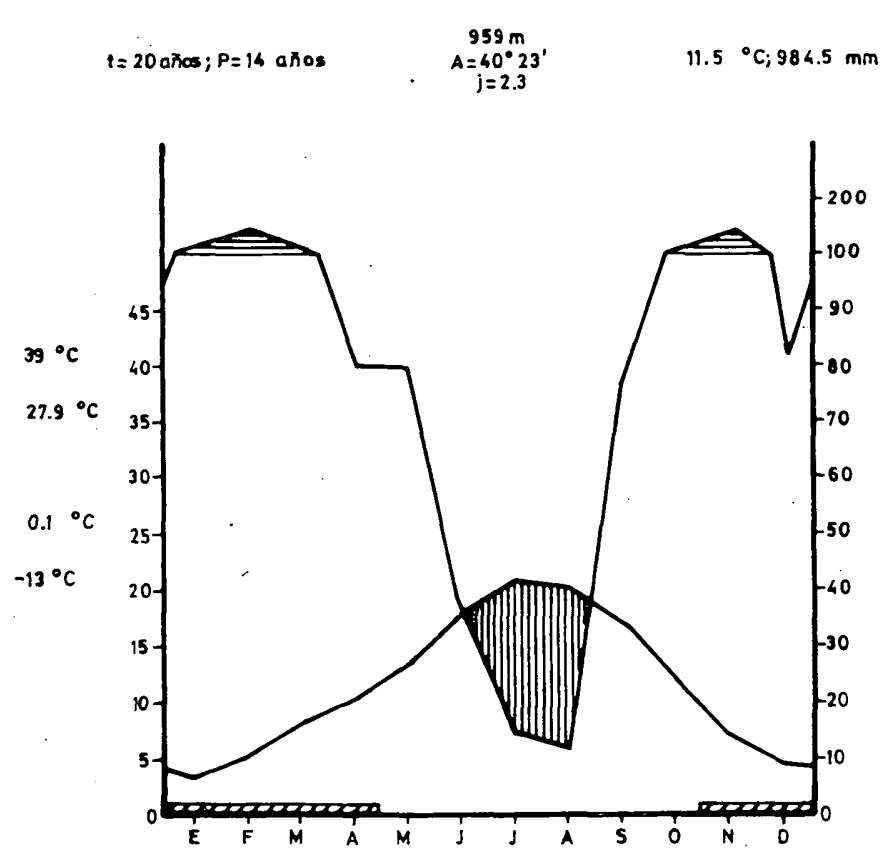

Fig. 2. Diagrama ombrotérmico de Walter-Gaussen para la zona de Béjar, con temperaturas de Barco de Avila corregidas (M.A.P.A., 1984). 
go, Rivas-Martínez (1979), por encima de los $1200 \mathrm{~m}$ y dentro de nuestra zona de estudio, estima un ombroclima húmedo, perteneciente a la región mediterránea, y establece por ello una serie de vegetación cuya etapa de bosque es ya caducifolio (Quercion broteri, Aceri-Quercion faginae, Quercion robori-pyrenaicae).

Clasificando el clima con los criterios de Rivas-Martínez (1979), es calificable como supramediterráneo inferior con inviernos frescos, en el aspecto térmico y supramediterráneo húmedo, en el aspecto de precipitación.

\subsection{Métodos}

Para la recogida de muestras de agua de lluvia se dispusieron 6 pluviómetros (con una superficie de recolección de $70.9 \mathrm{~cm}^{2}$ ); se colocaron a distintas alturas, de tal forma que cuatro quedaban debajo de la canopia vegetal (estimación del pluviolavado o trascolación) y los otros dos sobrepasan la copa del árbol (estimación del agua incidente); cada pluviómetro estaba comunicado por un tubo de plástico a unos recipientes. instalados en el suelo con capacidad suficiente para que no rebase el agua durante períodos de intensa precipitación, antes de su medida (Santa Regina et al. 1987). El tiempo de recogida de muestras se extendió desde el 27 de abril de 1984 hasta el 6 de Noviembre de 1986. Además, el agua de escurrimiento fustal pudo cuantificarse gracias a la colocación de una chapa galvanizada dispuesta helicoidalmente alrededor del tronco de cinco árboles representativos de la zona cercada, según el método propuesto por Ford \& Deans (1978); la precipitación recogida de esta forma es conducida a un depósito de plástico de gran capacidad. Para el cálculo de sus valores, se ha estimado, al igual que la mayoría de los autores, de forma un tanto arbitraria, pero generalmente aceptada, una corona circular, rodeando la base del tronco y con un radio máximo de 30 $\mathrm{cm}$ superior al del árbol, como superficie base sobre la que estimar los aportes del agua de escurrimiento por unidad de superficie; Mina (1967) limita el efecto a un radio de acción de $30-50 \mathrm{~cm}$ y a una profundidad de $1 \mathrm{~m}$, alrededor del tronco.

También se ha calculado el agua interceptada por diferencia entre el agua incidente $y$ el pluviolavado $y$ escurrimiento fustal, mediante la siguiente fórmula

$$
I=P-(T+F)
$$

Donde I: Agua interceptada

$P$ : Lluvia incidente

T: Trascolación o pluviolavado

F: Escurrimiento fustal 
Se ha empleado el método estadístico LSD (mínimas diferencias significativas), no apreciándose diferencias significativas entre los pluviómetros colocados, tanto los de encima como los de debajo de la cobertura vegetal del árbol; sin embargo, sí se han estimado diferencias para el dispositivo $n^{\circ} 4$ colocado alrededor del árbol.

Tan pronto como fue posible, se realizó el análisis de bioelementos; se tomaron alicuotas de la muestra de agua previamente concentrada y, con el fin de destruir la materia orgánica y poner en solución los metales presentes en la muestra, se sometieron a un proceso de digestión con $\mathrm{HNO}^{3}$, aquellas destinadas a la determinación de calcio, magnesio, sodio, potasio, hierro, manganeso, cobre y zinc; para la determinación del fósforo, se empleó digestión con $\mathrm{HClO}^{4}$, oxidando la materia orgánica y liberando fósforo como ortofosfato (Standard Method, 1980), procediéndose a su análisis por colorimetría, con metavanadato amónico (Chapman y Pratt, 1979; Standard Methods, 1980); el carbono total se determinó por vía húmeda mediante un analizador T.O.C.A. 915 A Beckman; el nitrógeno total mediante microkjeldahl Bouat-Afora; potasio y sodio, en un fotómetro de llama Flapho 4; calcio, magnesio, hierro, manganeso, cobre y zinc por absorción, en un espectrofotométro de absorción atómica Varian 1200.

\section{Resultados y discusión}

\subsection{Distribución del agua de lluvia}

La cantidad de lluvia incidente, agua de pluviolavado y escurrimiento fustal del bosque estudiado se representan en forma acumulativa para cada anualidad considerada en la Fig. 3.

Se pueden establecer dos ciclos completos de lluvia: uno, desde mediados de noviembre de 1984 hasta la misma época en 1985; y otro, desde esta última fecha hasta el 6 de noviembre de 1986 (Santa Regina et al., 1989).

Como resultado se ha de reseñar que durante el primer ciclo se recogen $1052 \mathrm{~mm}$ de lluvia incidente (de los cuales percolan $835 \mathrm{~mm}$ a través del dosel arbóreo) y $1.021 \mathrm{~mm}$ durante el segundo (percolando esta vez 824 $\mathrm{mm}$ a través de la canopia vegetal, y $110 \mathrm{~mm}$ que escurren a lo largo del tronco), por lo que en este $2^{\circ}$ ciclo se han estimado $91 \mathrm{~mm}$ de agua interceptada. Los resultados de este $2^{\circ}$ ciclo se representan más detalladamente en la figura 4, mostrándose en la tabla 1 los porcentájes para cada fracción hídrica considerada (Santa Regina et al. 1989).

Considerando la lluvia incidente (Fig. 3), se observan máximos entre los meses de abril/mayo (360 $\mathrm{mm}$ en abril de 1985, $265 \mathrm{~mm}$ en mayo de 1984; $154 \mathrm{~mm}$ en mayo de 1986), y otro en el inicio del otoño (355 mm en noviembre de 1985; $201 \mathrm{~mm}$ en octubre de 1986). 


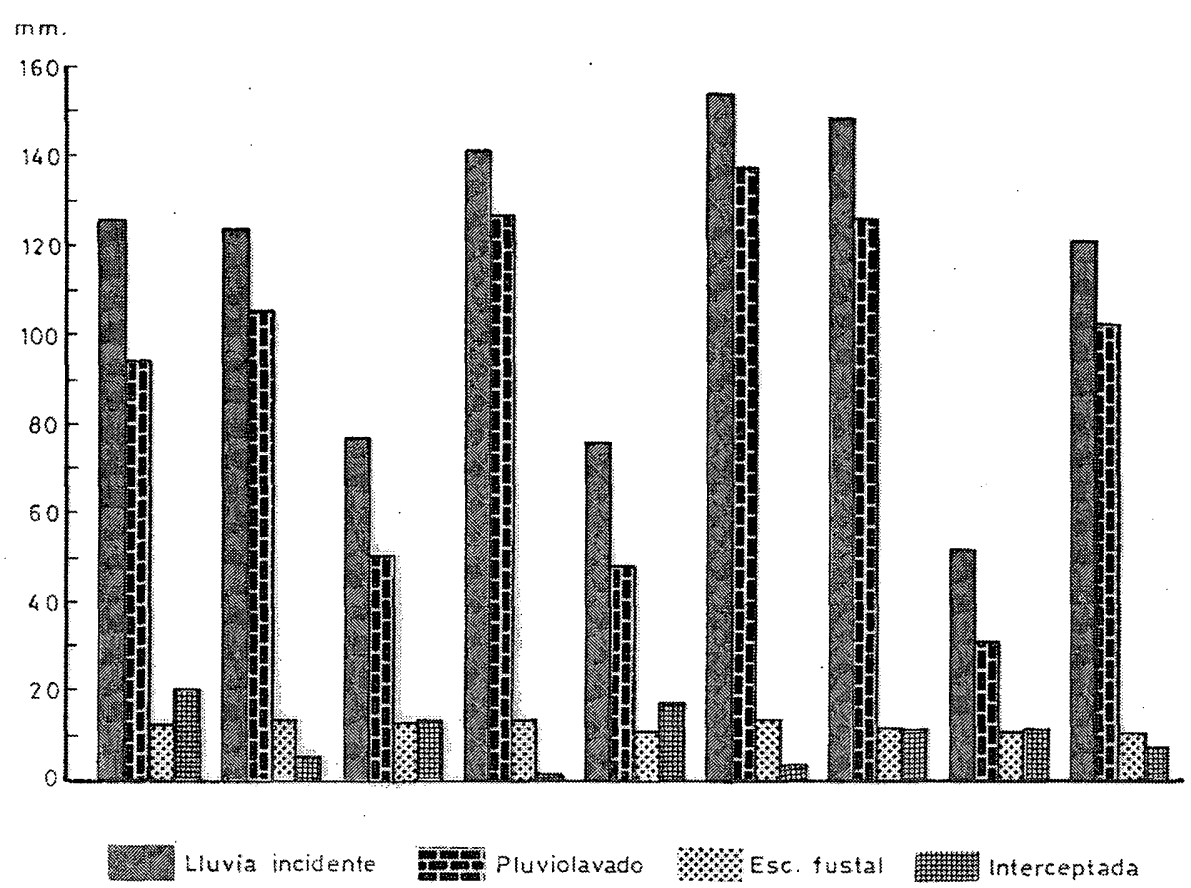

Fig. 3. Evolución de la lluvia incidente, pluviolavado y escurrimiento fustal estimada durante el periodo de observación.

Concretándose al segundo ciclo pluviométrico (Tabla 1), se observa que el porcentaje medio de pluviolavado es del $80 \%$, oscilando entre el $63 \%$ (abril) y el $90 \%$ (marzo), oscilación que no parece correlacionarse con la temperatura ambiente, sino más bien con otras variables (v.g.: viento, distribución diaria de lluvias), puesto que una vez que la capa foliar se satura de agua, su capacidad de intercepción disminuye drásticamente. Rapp y Romane (1968) estiman que las diferencias de porcentaje de pluviolavado entre una especie y otra son, principalmente, función de la intensidad y duración de las lluvias incidentes, dando un valor medio de $78.5 \%$ en Pinus halepensis; un valor algo inferior a éste establecieron Will (1959) en $P$. radiata (71\%), Rapp $\&$ Ibrahim (1978) en $P$. pinea deducen el $70 \%$ y Stogsdill et al. (1989) en $P$. taeda calculan alredor del $91 \%$.

De los $1021 \mathrm{~mm}$ de precipitación total (tabla 1) en este segundo ciclo, $110 \mathrm{~mm}$ escurren a lo largo de ramas y tronco (Fig. 3), que equivale a un porcentaje medio del $11.9 \%$, oscilando entre el 8 al $21 \%$ en un mismo mes (octubre de 1986), probablemente, también dependiendo del régimen de lluvias. Estas grandes variaciones se encuentra también en la bibliografía; 


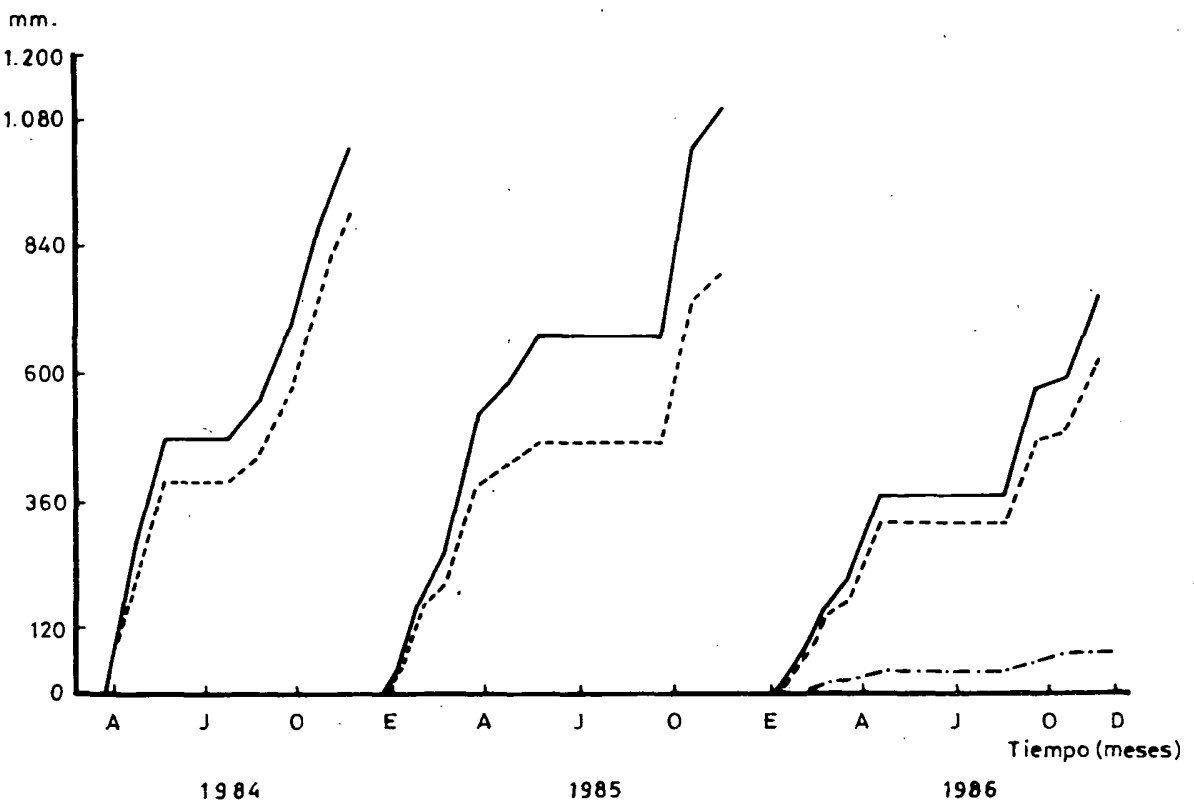

Fig. 4. Repartición del agua estimada según los muestreos del último ácido.

Tabla 1. Porcentaje del agua de pluviolavado, escurrimiento fustal e intercepción, con respecto a la l/uvia incidente total (Santa Regina et al. 1989)

\begin{tabular}{|c|c|c|c|c|}
\hline Fecha & $\begin{array}{c}\text { Lluvia } \\
\text { Incidente } \\
\text { P (mm) }\end{array}$ & $\begin{array}{c}\text { Pluviolavado } \\
\% \mathrm{~T}\end{array}$ & $\begin{array}{c}\text { Escurrimiento } \\
\text { fustal } \\
\% \mathrm{~F}\end{array}$ & $\begin{array}{c}\text { Intercepción } \\
\text { (calculada) } \\
\% \text { I }\end{array}$ \\
\hline $14-11-1985$ & 126.2 & 74.2 & 9.7 & 16.0 \\
$29-11-1985$ & 124.0 & 85.0 & 10.7 & 4.3 \\
$11-12-1985$ & 76.9 & 65.5 & 16.7 & 17.8 \\
$03-03-1986$ & 141.0 & 89.9 & 9.6 & 0.6 \\
$14-04-1986$ & 76.2 & 63.1 & 14.4 & 22.4 \\
$13-05-1986$ & 154.4 & 88.8 & 8.9 & 2.3 \\
$07-10-1986$ & 148.9 & 84.4 & 20.5 & 7.7 \\
$23-10-1986$ & 52.6 & 58.4 & 8.6 & 21.1 \\
$06-11-1986$ & 120.8 & 85.0 & $\times=11.9$ & $\times=11.0$ \\
& 1.021 .0 & $\times=77.2$ & $\times=1.9$ \\
\hline
\end{tabular}


asi, Aussenac (1968) en pinos y abetos encuentra valores muy bajos de escurrimiento fustal (1-2\%); Ford \& Deans (1978) lo cifran en un $27 \%$ en Picea sitchensis, mientras que Rapp \& Ibrahim (1978) en Pinus pinea, tan sólo en un 2-3\% y Gash y Morton (1978) en Pinus sylvestris alrededor del $1.6 \%$, por lo que se puede establecer que la morfología, edad, manejo,etc., del bosque influye grandemente en estos resultados.

El agua de intercepción calculada durante el $2^{\circ}$ ciclo (Tabla 1 ) alcanza un porcentaje medio del $9 \%$, oscilando inversamente el agua de pluviolavado (desde tan sólo un 0,6\% en marzo hasta un $22 \%$ en abril de 1986); esta gran variación se observa también en la bibliografía, y así Huber y Oyarzun (1983) estiman valores entre el 4 y el $53 \%$ en bosques de Pinus radiata, aunque el valor medio no alcanza el $10 \%$, cuando la precipitación continua es superior a $40 \mathrm{~mm}$; por otra parte, Aussenac (1968) estima que la intercepción es importante en coníferas, ya que establece valores del $30 \%$ en Pinus sy/vestris, del $34 \%$ en Picea abies y del $42 \%$ en Abies grandis, dando valores más bajos para caducifolios, por causa de su morfología. En Pinus patula, Schulze et al. (1978) estiman alrededor de un $30 \%$. Correlacionando P (pluviometría) I (intercepción), resulta la ecuación de regresión siguiente, significativa $(P>0.99)$ :

$$
\mathrm{I}=33.6-0.2 \mathrm{P}(\mathrm{r}=0.86 ; \mathrm{n}=9)
$$

lo cual confirma que a menor intensidad de lluvia, mayor intercepción (Santa Regina et al. 1989).

\subsection{Composición química del agua de lluvia}

Analizando los resultados de la composición química mineral (Tabla 2) del agua que incide hacia el substrato edáfico del pinar, se establecen las siguientes sucesiones:

Lluvia incidente .... C $>\mathrm{N}>\mathrm{Ca}>\mathrm{Mg}-\mathrm{k}-\mathrm{Zn}>\mathrm{Na}>\mathrm{P}-\mathrm{Fe}-\mathrm{Mn}>\mathrm{Cu}$

Pluviolavado ....... C $>\mathrm{N}>\mathrm{K}>\mathrm{Ca}>\mathrm{Zn}>\mathrm{Mg}-\mathrm{Na}-\mathrm{Mn}>\mathrm{Fe}-\mathrm{P}>\mathrm{Cu}$

Escurrimiento fustal. $\mathrm{C}>\mathrm{N}>\mathrm{K}>\mathrm{Ca}>\mathrm{Mn}>\mathrm{Mg}-\mathrm{Fe}>\mathrm{Na}-\mathrm{Zn}>\mathrm{P}>\mathrm{Cu}$

(téngase en cuenta que son resultados de análisis efectuados en aguas recogidas en Invierno de 1985-1986).

Repasando la contribución de cada bioelemento se observa que la concentración de carbono es superior en el agua de pluviolavado, lo que significa un arrastre de ácidos orgánicos excretados por las hojas y arrastrados por lavado; dichos ácidos están muy poco estudiados, aunque ciertos autores determinan los constituyentes orgánicos por lavado artificial de la superficie foliar (Dormaar , 1970); de todas formas son bajos los porcentajes de carbono puestos en circulación a través del agua de lluvia, 13 ppm en el pluviolavado, 7 ppm (Tabla 2) en lluvia incidente y 8 ppm en escurrimiento fustal (Santa Regina et al. 1989). 
PIRINEOS $145-146$

Tabla 2. Composición química del agua de lluvia (invierno de 1986). (Santa Regina 1987)

\begin{tabular}{|l|c|c|c|c|c|c|c|c|c|c|c|c|}
\hline $\begin{array}{l}\text { Elementos } \\
\text { (mg/Kg) }\end{array}$ & $\mathrm{C}$ & $\mathrm{N}$ & $\mathrm{Ca}$ & $\mathrm{Mg}$ & $\mathrm{P}$ & $\mathrm{K}$ & $\mathrm{Na}$ & $\mathrm{Fe}$ & $\mathrm{Mn}$ & $\mathrm{Cu}$ & $\mathrm{Zn}$ & $\mathrm{C} / \mathrm{N}$ \\
\hline $\begin{array}{l}\text { Lluvia incidente } \\
\text { Pluviolavado } \\
\begin{array}{l}\text { Escurrimiento } \\
\text { fustal }\end{array}\end{array}$ & $\begin{array}{c}6.75 \\
12.80\end{array}$ & 0.72 & 0.18 & 0.10 & 0.012 & 0.10 & 0.05 & 0.01 & 0.01 & 0.0006 & 0.10 & 9.3 \\
\hline
\end{tabular}

El nitrógeno, al igual que el carbono, se concentra preferentemente en pluviolavados, con un porcentaje de 1,6 ppm (Tabla 2), siendo algo superior at del escurrimiento fustal (1-ppm).

Aunque en pequeñas proporciones, sobresale el alto contenido de potasio en el agua de pluviolavado y escurrimiento fustal, comparado con el del agua incidente, debido a la eliminación a través de los órganos foliares y leñosos de este elemento, por secreción de los órganos aéreos; sin embargo, en las precipitaciones incidentes su concentración es similar a la de magnesio y zinc.

Es de resaltar un lugar destacado relativamente para el zinc en el agua de pluviolavado, al producirse secrecciones foliares importantes de este elemento, ya que en nuestros bosques hay un alto consumo de zinc, liberado por la roca madre original; también hay enriquecimiento de magnesio en el éscurrimiento fustal.

El cobre es el elemento mineral analizado que en menor proporción contiene el agua de lluvia; su contribución es prácticamente inexistente.

Asimismo, es de destacar la escasa concentración de fósforo, ocupando los últimos lugares de las sucesiones, al igual que los oligoelementos.

\subsection{Aporte de bioelementos por las precipitaciones}

El aporte medio estimado de elementos minerales al suelo del pinar por las precipitaciones se indica en la Tabla 3; en ella puede observarse que la cantidad de dichos elementos que alcanza al medio edáfico por medio del agua incidente y de pluviolavado es prácticamente idéntica durante los dos ciclos de experimentación, por lo que puede estimarse su media.

Se ha determinado un aporte al suelo del bosque de $70 \mathrm{Kg} / \mathrm{ha}$ de carbono total por medio de las precipitaciones incidentes, $107 \mathrm{Kg} / \mathrm{ha}$ por pluviolavados y $8,9 \mathrm{Kg} / \mathrm{ha}$ por escurrimiento fustal, (Tabla 3) valores inferiores a los obtenidos por Carlisle et al. (1966) bajo cubierta de Quercus petraea; el alto contenido de carbono en aguas de pluviolavados puede atribuirse a la presencia de un cierto número de ácidos alifáticos y ácidos fenoles libres (Bruckert et al., 1971). 
Tabla 3. Aportes de bioelementos al suelo por el agua ( $\mathrm{kg} / \mathrm{ha}$ ). (Santa Regina 1987)

\begin{tabular}{|c|c|c|c|c|c|c|c|c|c|c|c|c|}
\hline Tiempo & Reparto & c & N & $\mathrm{Ca}$ & $\mathrm{Mg}$ & $P$ & K & $\mathrm{Na}$ & $\mathrm{Mn}$ & $\mathrm{Fe}$ & $\mathrm{Cu}$ & $\mathrm{Zn}$ \\
\hline \multirow{2}{*}{$\begin{array}{l}\text { Desde } \\
16-11-1984 \\
\text { a } \\
\text { 04-11-1985 }\end{array}$} & $\begin{array}{l}\text { Lluvia incidente } \\
(1.052 \mathrm{~mm})\end{array}$ & 71.0 & 7.6 & 1.9 & 1.1 & 0.13 & 1.1 & 0.53 & 0.11 & 0.11 & $6.3 \times 10^{-3}$ & 1.1 \\
\hline & $\begin{array}{l}\text { Pluviolavado } \\
(835 \mathrm{~mm})\end{array}$ & 106.9 & 13.4 & 2.5 & 0.9 & 0.30 & 5.8 & 0.75 & 0.67 & 0.33 & $5.0 \times 10^{-3}$ & 1.7 \\
\hline \multirow{6}{*}{$\begin{array}{l}\text { Desde } \\
\text { 14-11-1985 } \\
\text { a } \\
\text { 06-11-1986 }\end{array}$} & $\begin{array}{l}\text { Lluvia incidente } \\
(1.021 \mathrm{~mm})\end{array}$ & 68.9 & 7.4 & 1.8 & 1.0 & 0.12 & 1.0 & 0.51 & 0.10 & 0.10 & $6.1 \times 10^{-3}$ & 1.0 \\
\hline & $\begin{array}{l}\text { PLuviolavado } \\
\text { (824 mm) }\end{array}$ & 105.5 & 13.2 & 2.5 & 0.9 & 0.29 & 5.80 & 0.74 & 0.66 & 0.33 & $4.9 \times 10^{-3}$ & 1.6 \\
\hline & & 92 & 93 & 86 & 82 & 94 & 87 & 85 & 69 & 67 & 39 & 94 \\
\hline & $\begin{array}{l}\text { fustal } \\
(110 \mathrm{~mm})\end{array}$ & 8.9 & 1.0 & 0.4 & 0.2 & 0.02 & 0.83 & 0.13 & 0.30 & 0.16 & $7.8 \times 10^{-3}$ & 0.1 \\
\hline & $\%$ & 8 & 7 & 14 & 18 & 6 & 13 & 15 & 31 & 33 & 61 & 6 \\
\hline & $\begin{array}{l}\text { Total aportado } \\
\text { al suelo }\end{array}$ & 114 & 14.2 & 2.9 & 1.1 & 0.31 & 6.63 & 0.87 & 0.96 & 0.49 & 0.013 & 1.7 \\
\hline
\end{tabular}


En cuanto a los demás elementos minerales, destaca el importante aporte de nitrógeno al suelo bajo forma de pluviolavado. Sobre esta cuestión destacan los estudios realizados por Grunert (1964), Lemeé \& Bichaut (1971); Lemeé (1974) y Mayer \& Ulrich (1974) en bosques de hayedos; Villecourt \& Roose (1978) en la savana, Nihlgard (1970), Lemeé (1974) en bosques de Fagus sylvatica, Carlisle et al. (1966) en bosques de Quercus petraea, Rapp (1969) en Quercus ilex, así como los de Nye (1961) en la selva tropical. La cantidad media de nitrógeno mineral aportado por las aguas de lluvia al subsistema edáfico se estima en $7,5 \mathrm{Kg} / \mathrm{ha}$ por lluvia directa, $13,3 \mathrm{Kg} / \mathrm{ha}$ por pluviolavados y $1 \mathrm{Kg} / \mathrm{ha}$ por escurrimiento fustal. Estos valores son similares a los de Rapp (1969), algo superiores a los estimados por Carlisle et al. (1967), Nihlgard (1970) y Lemeé (1974), algo inferiores a los de Anderson (1973) y muy inferiores a los de Villecourt 8 Roose (1978) y Ranger \& Nys (1986).

Por medio de las aguas de lluvia, el suelo del pinar se beneficia de un aporte en calcio de $1,9 \mathrm{Kg} / \mathrm{ha}$ por precipitaciones incidentes, $2,5 \mathrm{~kg} / \mathrm{ha}$ por pluviolavados y $0,4 \mathrm{Kg} / \mathrm{ha}$ por escurrimiento fustal; valores similares a los establecidos por Lemeé (1974) y muy inferiores a los de Nihlgard (1970) y Villecourt \& Roose (1978).

Puede establecerse un empobrecimiento de calcio en el medio edáfico por lavado de este elemento hacia las capas subyacentes; en condiciones naturales, es probable que las raíces absorban una gran cantidad de calcio móvil antes de su lavado (Rapp, 1971).

Destaca el hecho de que el suelo del bosque se beneficia de un aporte en magnesio por precipitaciones directas, similar al obtenido por pluviolavados; se estiman $1,1 \mathrm{Kg} / \mathrm{ha}$ aportado por las lluvias directas, 0,9 $\mathrm{kg} / \mathrm{ha}$ por pluviolavados (no estimándose diferencias significativas) y 0,2 $\mathrm{kg} /$ ha por escurrimiento fustal; estos valores son inferiores a los obtenidos por otros autores (Rapp, 1971); Aussenac et al., 1972; Lemeé, 1974; Villecourt \& Roose, 1978 y Ranger $\&$ Nys, (1986); Rapp (1971) en bosques de Quercus ilex estima que el magnesio existe en cantidad superior a la puesta en circulación, siendo susceptible de ser eliminado por pluviolavado.

Son muy escasas las cantidades de fósforo que retornan al suelo del bosque por medio de las precipitaciones $(0,13 \mathrm{~kg} / \mathrm{ha}$ por lluvia incidente, $0,3 \mathrm{Kg} / \mathrm{ha}$ por pluviolavados y $0,02 \mathrm{Kg} / \mathrm{ha}$ por escurrimiento fustal); valores similares a los establecidos por Aussenac et al. (1972), Lemeé (1974) y Ranger \& Nys (1986); inferiores los de Rapp (1971) y Nihlgard (1970) y muy inferiores a los de Villecourt \& Roose (1978).

En cuanto a la cantidad de potasio que alcanza el suelo hay que resaltar los $0,8 \mathrm{Kg} / \mathrm{ha}$ por escurrimiento a lo largo del tronco y $5,8 \mathrm{~kg} / \mathrm{ha}$ por pluviolavado. Rapp (1971) establece una cantidad de $28 \mathrm{~kg} / \mathrm{ha}$, aunque indica que $26 \mathrm{~kg} / \mathrm{ha}$ pueden provenir de secreciones foliares por eliminación a través de la corteza o por el lavado y arrastre de depósito y restos diversos, mientras que los otros $2 \mathrm{~kg} / \mathrm{ha}$ estarían contenidos en las lluvias incidentes. En el substrato edáfico, el potasio es rápidamente arrastrado 
por las aguas de infiltración desde los $10 \mathrm{~cm}$ superficiales hacia los horizontes inferiores, donde va a ser fijado por el complejo absorbente, reabsorbido por las raices (Remezov \& Pogreanyak, 1969).

El suelo del pinar se beneficia de un aporte en sodio de $0,5 \mathrm{~kg} / \mathrm{ha}$ por medio de las precipitaciones incidentes; $0,8 \mathrm{~kg} / \mathrm{ha}$ por pluviolavados $y$ $0,13 \mathrm{~kg} / \mathrm{ha}$ por escurrimiento fustal. Este alcalino es muy soluble y debe percolar a los horizontes inferiores e, incluso, ser arrastrado fuera del ecosistema, como ya lo ha constatado Rapp (1971); los valores obtenidos por otros autores son muy superiores a los estimados en el presente trabajo (Nihlgard,1970; Rapp, 1971; Villecourt \& Roose, 1978).

Son escasas las cantidades de oligoelementos que alcanzan el substrato edáfico por medio del agua de lluvia, destacando la alta proporción de zinc en pluviolavados, superior incluso a la de magnesio, sodio y fósforo; dicha proporción puede atribuirse al lavado de secreciones cuticulares, al ser este elemento abundante en nuestra zona de estudio. La contribución de cobre al aporte total de oligoelementos al suelo es prácticamente despreciable.

El aporte de hierro y manganeso por precipitaciones incidentes es similar; sin embargo, por pluviolavados y escurrimiento fustal se arrastra mayor cantidad de manganeso, probablemente bajo forma valencia dos (solubles).

\subsection{Repartición de bioelementos}

Consecuentemente, las aguas de lluvia recogidas bajo cubierta forestal pueden aportar al suelo, por lavado de las partes aéreas del árbol, una cantidad a veces considerable de elementos del árbol. Este aporte puede ser tan importante como áquel que proviene del retorno anual de órganos vegetales (Denaeyer-De Smet, 1966; Rapp, 1971).

Las precipitaciones sobre el dosel arbóreo del pinar estudiado se distribuyen en pluviolavados, agua de escurrimiento fustal y agúa interceptada, por lo que no deben considerarse aportes de bioelementos por agua libre debido a la alta densidad de árboles (alrededor de 1.700/ha, esto es, las copas de dichos árboles se encuentran prácticamente en contacto), lo cual no permite entrada importante de aquélla.

Por tanto, la cantidad anual de bioelementos que alcanza el suelo del bosque se establece (suma de pluviolavado más escurrimiento), de la forma siguiente (Tabla 3): $114 \mathrm{~kg} / \mathrm{ha}$ de carbono total, $14 \mathrm{~kg} / \mathrm{ha}$ de nitrógeno, $3 \mathrm{~kg} / \mathrm{ha}$ de calcio, $1 \mathrm{~kg} / \mathrm{ha}$ de magnesio, $0,3 \mathrm{~kg} / \mathrm{ha}$ de fósforo, 7 $\mathrm{kg} / \mathrm{ha}$ de potasio, $0,9 \mathrm{~kg} / \mathrm{ha}$ de sodio, $1 \mathrm{~kg} / \mathrm{ha}$ de manganeso, $0,5 \mathrm{~kg} / \mathrm{ha}$ de hierro, $12 \mathrm{~g} / \mathrm{ha}$ de cobre y $2 \mathrm{Kg} / \mathrm{h} a$ de zinc. 


\section{Conclusiones}

a) Se ha estimado que cada año el suelo del bosque se enriquece en varios $\mathrm{kg} / \mathrm{ha}$ de macroelementos (sobre todo nitrógeno, potasio y calcio) procedentes del agua de lluvia (escurrimiento fustal + trascolación); en cuanto a los oligoelementos, los aportes por el agua de lluvia (que incluye polvos atmosféricos) pueden ser superiores incluso a la contribución de la propia hojarasca.

b) Fundamentalmente, se aportan por pluviolavado (más del $80 \%$ del total que accede al suelo) carbono, nitrógeno, calcio, magnesio, fósforo, potasio, sodio y zinc; en su mayor parte (entre el 60 y el $80 \%$ ) el manganeso y el hierro, aunque hay que considerar, por ser significativo, el escurrimiento fustal; por el contrario, el cobre procede mayoritariamente del escurrimiento fustal aunque en escasa cantidad (del orden de $0.01 \mathrm{Kg} /$ ha.).

\section{Referencias}

Anderson J.M. (1973): The breakdown and decomposition of sweet chesnut (Castanea sativa Mill) and beech (Fagus sylvatica L.) leaf litter in two deciduous woodland soils. I. Breakdown leaching and decomposition. Oecología. 12: 251 274.

Aussenac G. (1968): Interception des précipitations par le couvert forestier. Ann. Sci. Forest. 25:135-156.

Aussenac, G. Bonneau M., Le Tacon F. (1972): Restitution des éléments minéraux au sol par l’intermediaire de la litiére et des précipitations dans quatre peuplements forestiers de l'est de la France. Oecol. Plant. 7: 1-21.

Bruckert S., Toutain F., Tchicaya J., Jacquin F. (1971): Influence des pluviolessivats de hêtre et de pin silvestre sur les processes d'humification. Oecol. Plant. 6: 329339.

Carlisle A., Brown A.H.F., White E. J. (1966): The organic mater and nutrient elements in the precipitations beneath a sessile oak canopy. J. Ecol. 54: 87-98.

Chapman H.D., Pratt P.F. (1979): Métodos de análisis de suelos, plantas yaguas. Trillas. México $195 \mathrm{pp}$.

Denaeyer-De Smet S. (1966): Bilan annuel des apports d'éléments minéraux dans les eaux de précipitation sous couvert forestier dans la forêt melangée caducifoliée de Blaimont. Bull. Soc. Roy. Bot. Belgique. 99: 345-375.

Diem H.G. (1973): Recherche sur la phyllosphére de l'orge. Thése de Doctorat d'Etat. Université de Nancy. $124 \mathrm{pp}$.

Dormaar J.F. (1970): Seasonal pattern of water soluble constituents from leaves of Populus northwes (Hort.) J. Soil Sci. 21:105-110.

F.A.O. (1973): The legend F.A.O./U.N.E.S.C.O.: Soil map of the world. F.A.O. Roma.

Ford E.D., Deans J.D. (1978): The effects of canopy structure on stemflow, throughfall and interception loss in a young sitka spruce plantation. J. App/. Ecol. 15: 905-917.

Gash J.H.C:, Morton A.J. (1978): An application of the Rutter model to the estimation of the interception loss from Thetford forest. J. Hydrol. 38: 49-59. 
Huber A., Oyarzún C. (1983): Factores reguladores de la intercepción en un bosque adulto de Pinus radiata (D. Don). Bosque 21 : 59-64.

Jackson I. J. (1975): Relationships between rainfall parameters and interception by tropical forest. J. Hydrol. 24: 215-238.

Lemée G. (1974): Recherches sur les écosystémes des reserves biologiques de la forêt de Fontainebleau. IV. Entrées d'éléments minéraux par les précipitations et transfer au sol par le pluviolessivage. Oecol. Plant. 9: 187-200.

Lemée G., Bichaut N (1971): Recherches sur les écosystémes des reserves biologiques de la forêt e Fontainebleau. I. Production de litiére. Oecol. Plant. 6 : 133-149.

Mayer R., Ulrich B. (1974): Conclusions on the filtering action of forests from ecosystem analysis. Oecol. Plant. 9: 157-168.

Nihlgard B. (1970): Precipitation, its chemical composition and effect on soil water in a bëech on an spruce forest in south sweeden. Oikos. 21:208-217.

Nihlgard B. (1972): Plant biomass, primary production and distribution of chemical elements in a beech and a planted spruce forest in south sweden. Oikos. 23: 69-81.

Nye P.H. (1961): Organic matter and nutrient cycles under moist tropical forest. $P$. Soil. 13: 333-346.

Patric J.H. (1966): Rainfall interception by mature coniferous forest of Southeast Alaska. J. Soil and water Conservation. 21: 229-231.

Ranger J., Nys C. (1986): Etude des éléments minéraux dans un taillis des Ardennes (Nord de la France): bilan pour une rotation compléte. Acta Oecol. Plant. 7: 287-305.

Rapp M. (1969): Apports d'éléments minèraux au sol par les eaux de pluviolessivage sous des peuplements de Quercus ilex L. Quercus lanuginosa Lam K et Pinus halepensis Mill. Oecol. plant. 4: 325-338.

Rapp M. (1971): Cycle de la matiére organique et des eléments mineraux dans quelques écosystéms méditerranéens. C.N.R.S. París. 184 pp.

Rapp M., Ibrahim M. (1978): Egouttement, écuolement et interception des précipitations par un peuplement de Pinus pinea L. Oecol. Plant. 13: 321-330.

Rapp M., Romane F. (1968): Contribution a l'étude du bilan de l'eau dans les écosystémes méditerranéens. 1. Egouttement des précipitaions sous des peuplements de Quercus ilex L. et de Pinus halepensis. Mill. Oecol. Plant. 3 : $271-284$

Remezov N.P. Pogrenyak P.S. (1969): Forest Soil Science. I.P.S. T. Press. Jerusalen. $261 \mathrm{pp}$

Rivas-Martínez (1979): Brezales y jarales de Europa occidental. Lazaroa. 1. Depart. de Bot. Facultad de Ciencias. Univ. Complutense. Madrid. 1: 5-128.

Rutter A.J. (1963): Studies in the water relations of Pinus sylvestris in plantation conditions. I measurement of rainfall and interception. Ecol. 51: 191-204.

Santa Regina I. (1987): Contribución al estudio de la dinámica de materia orgánica y bioelementos en bosques de la Sierra de Béjar. Tesis doctoral. Univ. de Salamanca. 464 pp.

Santa Regina I., Gallardo J.F. (1989): Biogeochemical cycles in forests of the Sierra de Béjar (Salamanca, Spain): return of bioelements in rainfall. Acta Oecologica. 10: 433-438.

Santa Regina 1., Gallardo J.F., San Miguel C., Moyano A. (1989): Intercepción, pluviolavado y escorrentía cortical en una plantación de Pinus sylvestris de la Cuenca de Candelario. (Centro-Oeste de España). Bosque. 10: 19-27. 
Santa Regina I., San Miguel C., Gallardo J.F. (1987): Estudio del balance de agua en ecosistemas forestales del Sistema Central: Pluviolavado, escurrimiento fustal e intercepciónen un bosque disclimácico de Pinus sylvestris L. VIII. Bienal de la R. Soc. Esp. de Historia Natural. Pamplona. 583-593.

Schnock G., Galoux A. (1967): Réception des precipitations et égouttement. Bull. Inst. Sci. Nat. Belg. 43: 1-30.

Schulze R.E., Scott C.R., Nanni U.V. (1978): Interception by Pinus patula in relation to rainfall parameters. J. Hydrol. 36: 393-396.

Slatyer R.O. (1962): Meassurement of precipitation interception by an arid zone plant community (Acacia aneura F. Muell.). Méthodologie en Ecophysiologie Végétale. ECKARDT, F.E. (ed). Actes colloque de Montpellier. UNESCO: 181192.

Slavik B. (1962): Rain interception in deciduous forest. Recherches sur la zone aride. Methodologie en Ecophysiologie Végétale. ECKARDT, F.E. (ed.)., Actes colloque de Montpellier. UNESCO: 193-199.

APHA. AWWA-WPCF (1980): Standard methods for the examination of water and wastewater. 1.134 pp. México.

Stogsdill W.R., Wittwer R.F., Hennessey T. C., Dougherty P.M. (1989): Relationship between throughfall an stand density in a Pinus taeda plantation. For. Ecol. and Manag. 29: 105-113.

Tukey H.B. (1966): Leaching of metabolites from above ground plant parts by leaching. Atompraxis. 6: 213-218.

Villecourt P., Roose E. (1978): Change en azote et en éléments minéraux majeurs des eaux de pluie, de pluviolessivage et de drainage dans la savanne de Lamto (côte d'lvoire). Rev. Ecol. Biol. Sol. 15: 1-20.

Will G.M. (1959): Nutrient return in litter and rainfall under some exotic-conifer stands in New Zealand. N.Z.J. Agr. Res. 2: 719-734 